\title{
On the Capacity of Low-Density Parity-Check Codes
}

\author{
Sae-Young Chung ${ }^{1}$ \\ Airvana, Inc. \\ 25 Industrial av. \\ Chelmsford, MA 01824 USA. \\ s-chung@airvananet.com
}

\begin{abstract}
We demonstrate that we can translate threshold values of low-density parity-check (LDPC) codes between channels accurately using a simple mapping. We develop some models of density evolution from this observation, namely erasurechannel and reciprocal-channel approximations. The reciprocal-channel approximation, based on dualizing LDPC codes, provides a very accurate model of density evolution for the additive-white Gaussian noise (AWGN) channel.
\end{abstract}

\section{INTRODUCTION}

Density evolution $[1,2]$ is a very useful tool to calculate the capacity of low-density parity-check (LDPC) codes under various decoding algorithms. LDPC codes with a threshold value within $0.0045 \mathrm{~dB}$ and a simulation result within 0.04 $\mathrm{dB}$ of the Shannon limit were demonstrated in $[4,5]$ using density evolution.

As shown in [4], the channel capacity of various binaryinput symmetric-output memoryless channels (binary-input channels from now on), including the binary erasure (BEC), binary symmetric (BSC), Laplace, AWGN channels, evaluated at the corresponding thresholds are very similar across these channels for various regular and irregular LDPC codes. This implies that the rate penalty for imposing an LDPC structure on optimal codes is similar across these channels.

One immediate application of this observation is that the calculation of thresholds for any binary-input channel can be carried out approximately (but reasonably accurately) by using the simplest channel, namely the BEC. We call this the "erasure-channel approximation."

This observation is also the key motivation for designing an improved model of density evolution for the AWGN channel, which we call the "reciprocal-channel approximation."

\section{Reciprocal-Channel Approximation}

We assume regular codes in this section. We first define a reciprocal-channel mapping $\psi_{\mathrm{X}}(\cdot): X \rightarrow X$ for the channel parameter $x \in X \subset \mathbb{R}$ of a binary-input channel $\mathrm{X}$ with its channel capacity $C_{\mathrm{X}}(x)$ (we assume this is monotonic in $x$ ) as

$$
\psi_{\mathrm{X}}(x)=C_{\mathrm{X}}^{-1}\left(1-C_{\mathrm{X}}(x)\right) .
$$

Note that the reciprocal-channel mapping is self-inverse. Using this definition, we define the reciprocal channel as follows.

Definition 1 The reciprocal channel of a channel $X$ with the parameter $x$ is the same channel with the parameter $\psi_{X}(x)$.

We define the reciprocal-channel approximation as a one-dimensional approximation to density evolution using

\footnotetext{
${ }^{1}$ This work is based on S.-Y. Chung's Ph.D. thesis [4].
}

\author{
G. David Forney, Jr. \\ Laboratory for Information and \\ Decision Systems, MIT, \\ Cambridge, MA 02139 USA. \\ forn:ey@lids.mit.edu
}

reciprocal-channel mappings, where all approximate messages are real numbers. To do this, we first find a mapping that maps the initial message density $p_{0}$ of a channel $\mathrm{X}$ to a real number $c_{0}$. We then find a symmetric computation rule $\tau(\cdot, \cdot)$ that maps $\mathbb{R}^{2}$ to $\mathbb{R}$. For a degree- $d_{v}$ variable node, this function is applied repeatedly to compute the $d_{v}$-th output message $b$ of the node, i.e.,

$$
b=\tau\left(a_{0}, \tau\left(a_{1}, \tau\left(\ldots, a_{d_{v}-1}\right) \ldots\right),\right.
$$

where $a_{1}, \ldots, a_{d_{v}-1}$ are the approximate output messages from check nodes from the previous iteration and $b=a_{0}$ in the initial iteration. Abusing notation, we denote the resulting mapping by $\tau(\cdot, \ldots, \cdot)$.

For check nodes, we define the computation rule using the reciprocal channel mapping $\psi_{\mathrm{X}}(\cdot)$, i.e.,

$$
a^{\prime}=\psi_{\mathrm{X}}\left(\tau\left(\psi_{\mathrm{X}}\left(b_{1}\right), \psi_{\mathrm{X}}\left(b_{2}\right), \ldots, \psi_{\mathrm{X}}\left(b_{d_{c}-1}\right)\right)\right),
$$

where the $b_{j}$ 's are inputs and $a^{\prime}$ is the $d_{c}$-th output of the check node. Note that the reciprocal channel mapping $\psi(\cdot)$ effectively dualizes the parity-check code.

We note that this approximation becomes exact for the BEC if the message is the erasure probability and $\tau\left(a_{1}, a_{2}\right)=$ $a_{1} a_{2}$.

For AWGN channels, we define the mean of the LLR message as the one-dimensional message and use $\tau\left(a_{1}, a_{2}\right)=$ $a_{1}+a_{2}$. This is about 10 times more accurate than the erasurechannel and Gaussian [3] approximations.

Live demonstration of density evolution and full papers are available at http://truth.mit.edu/ sychung.

\section{ACKNOWLEDGMENTS}

The first author would like to express his appreciation to Thomas J. Richardson and Rüdiger Urbanke for their many helpful comments and suggestions on this paper.

\section{REFERENCES}

[1] T. J. Richardson and R. Urbanke, "The capacity of low-density parity-check codes under message-passing decoding," IEEE Trans. Inform. Theory, vol. 47, pp. 599-618, Feb. 2001.

[2] T. J. Richardson, A. Shokrollahi, and R. Urbanke, "Design of capacity-approaching low-density parity-check codes," IEEE Trans. Inform. Theory, vol. 47, pp. 619-637, Feb. 2001.

[3] S.-Y. Chung, T. J. Richardson, and R. Urbanke, "Analysis of sum-product decoding of low-density parity-check codes using a Gaussian approximation," IEEE Trans. Inform. Theory, vol. 47, pp. 657-670, Feb. 2001.

[4] S.-Y. Chung, "On the construction of capacity-approaching coding schemes," Ph.D. dissertation, Mass. Inst. Technol., Cambridge, MA, 2000. Available at http://truth.mit.edu/ sychung.

[5] S.-Y. Chung, G. D. Forney, Jr., T. J. Richardson, and R. Urbanke, "On the design of low-density parity-check codes within $0.0045 \mathrm{~dB}$ of the Shannon limit," IEEE Commun. Lett., vol. 5, pp. 58-60, Feb. 2001 